\title{
Disfunção temporomandibular e hábitos parafuncionais em crianças e adolescentes
}

\author{
Temporomandibular dysfunction and habits parafunction in children and adolescents
}

Manoel Gomes de Araúio Neto', Alisson Sousa Santos ${ }^{1}$, Paulo Henrique Martins Sousa', Laryssa Castro Vale Caroline Fernanda de Oliveira Farias Lopes', Guilherme Gonçalves Silva Pinto², Ana Lurdes Avelar Nascimento³, Adelzir Malheiros e Silva C. B. Haidar ${ }^{3}$, Sarah Tarcísia Rebelo Ferreira de Carvalho ${ }^{3}$, Maria Claudia Gonçalves ${ }^{4}$

'Discente do Curso de Graduação em Fisioterapia da Universidade CEUMA. Aluno de iniciação científica do Núcleo de Pesquisa em Reabilitação Funcional e Atenção à Saúde/NUPERF/CEUMA/CNPQ ${ }^{2}$ Discente do Curso de Medicina das Faculdades Integradas da União Educacional do Planalto Central-FACIPLAC Membro do Núcleo de Pesquisa em Reabilitação Funcional e Atenção à Saúde/NUPERF/CEUMA/CNPQ ${ }^{3}$ Docente titular do Curso de Fisioterapia da Universidade CEUMA. Pesquisadora Membro do Núcleo de Pesquisa em Reabilitação Funcional e Atenção à Saúde/NUPERF/CEUMA/CNPQ ${ }^{4}$ Docente titular do Programa de Pós-graduação em Meio Ambiente e do Curso de Fisioterapia da Universidade CEUMA. Pesquisadora do Núcleo de Pesquisa Mecanismos de modulação de saúde e ambiente no Maranhão/CEUMA/CNPQ

Araújo Neto MG, Santos AS, Sousa PHM, Vale LC, Lopes CFOF, Pinto GGS, Nascimento ALA, Haidar AMSCB, Carvalho STRF, Gonçalves MC. Disfunção temporomandibular e hábitos parafuncionais em crianças e adolescentes. Headache Medicine. 2017;8(4):120-123

\section{RESUMO}

Introdução: A Disfunção temporomandibular (DTM) na maioria das vezes é descoberta durante a fase adulta, porém seus sintomas podem começar cedo, ainda na infância. Devido às grandes transformações musculares, esqueléticas e articulares presentes durante o crescimento craniofacial, crianças e adolescentes podem apresentam sinais e sintomas de DTM. Objetivo: Avaliar a frequência de sinais e sintomas e o diagnóstico da DTM, bem como os hábitos parafuncionais orais em crianças e adolescentes. Material e Método: Participaram deste estudo 44 crianças e adolescentes, regularmente matriculados na Escola U.E.B Luís Viana /Alemanha e na Escola Nosso Mundo/Bequimão situada na cidade de São Luís- MA, com idade entre 10 e 15 anos, foram excluídos aqueles indivíduos que tenham realizado cirurgias ortognáticas prévias, tenham feito ou estejam realizando tratamentos ortodônticos ou para DTM's, que tivessem sofrido trauma na face e que não apresentassem 0 consentimento assinado pelos pais. A severidade da DTM foi avaliada com o índice anamnésico de Fonseca e o diagnóstico de DTM com o Critério diagnóstico em pesquisa RDC/TMD. Este projeto foi aprovado pelo Comitê de Ética em pesquisa da Universidade CEUMA, parecer $n^{\circ} 1.307 .233$. Todos os pais e responsáveis assinaram o termo de Consentimento Livre e esclarecido. Resultados: $O$ diagnóstico de DTM em crianças e adolescentes foi demonstrado e o mes- mo se apresentou elevado. Os sinais e sintomas de DTM e os hábitos parafuncionais orais também apresentaram frequência elevada e podem ser usados como preditores do agravamento da DTM.

Palavras-chave: Disfunção temporomandibular; severidade; diagnóstico

\section{RESUMO}

Introduction: Temporomandibular dysfunction (DTM) Most of the time is discovered during the adult phase, but its symptoms may be ginearly, even in childhood. Due to large muscular, skeletal and joint transformations present during craniofacial growth, children and adolescents may present signs and symptoms of DTM. Objective: To evaluate the frequency of signs and symptoms and the diagnosis of DTM, as well as the oral parafunctional habits in children and adolescents. Material and Method: Participated in this study 44 children and adolescents, regularly enrolled in the EU school B Luís Viana/germany and at the schoolour World/Bequimão located in the city of São Luís-MA, aged between 10:15 years, were excluded those individuals who have performed preortognáticas surgeries, have done or are conducting or thodontic treatments or for DTM'S, who had suffered trauma to the face and did not present the consent signed by the parents. The severity of the DTM was evaluated with the anamnesico 
content of Fonseca and the diagnosis of DTM with the diagnostic criterion in research RDC/TMD. This project was approved by the CEUMA University Research Ethics Committee, Opinion No. 1,307,233. All parents and guardians signed the term of consent free and clear. Results: The diagnosis of DTM in children and adolescents was demonstrated and the same wa selevated. The signs and symptoms of DTM and the oral parafunctional habits also presented high frequency and can be used as predictors of the worsening of DTM.

Keywords: Temporomandibular dysfunction; Severity Diagnosis.

\section{INTRODUÇÃO}

Disfunção Temporomandibular (DTM) é um termo coletivo que envolve todas as condições relativas a Articulação Temporomandibular (ATM) e estruturas do sistema estomatognático ou mastigatórios associadas. A DTM se refere a um aglomerado de desordens caracterizadas por ruídos articulares, limitação na amplitude de movimento ou desvios durante a função mandibular, que são considerados como sinais de DTM, e dor pré-auricular, dor na ATM ou nos músculos mastigatórios, caracterizados como sintomas. ${ }^{(1)}$

A etiologia da DTM é multifatorial, entre os fatores etiológicos estão traumas da mandíbula ou ATM, maloclusão e interferências oclusais, alterações nos músculos mastigatórios, microtraumas causados pelos hábitos parafuncionais, condições reumáticas, estresse emocional, ansiedade e anormalidades posturais. ${ }^{(2)}$

A DTM geralmente é diagnosticada na fase adulta, entretanto já na fase infantil se pode perceber alguns sinais e sintomas. Na criança e no adulto com até 40 anos de idade prevalece a DTM do tipo miogênica, aquele em que somente os músculos estão envolvidos, e, após essa idade, o fator principal da etiologia é degenerativo, envolvendo além dos músculos a articulação (DTM artrogênica). ${ }^{(3)}$ Durante o período de crescimento, devido ao desenvolvimento craniofacial, existe a possibilidade do desenvolvimento significativo de sinais e sintomas de DTM, predispondo as crianças ao surgimento de tal distúrbio. ${ }^{(4)}$

Entre os fatores etiológicos, os hábitos parafuncionais orais parecem ser importantes para o surgimento da DTM em crianças. ${ }^{(3)}$ Dessa forma, a hipótese desse trabalho é que crianças e adolescentes apresentem elevada frequência de diagnóstico de DTM bem como de hábitos parafuncionais.

\section{Objetivo}

Avaliar a presença do diagnóstico da DTM e de hábitos parafuncionais orais em crianças e adolescentes.

\section{MATERIAL E MÉTODOS}

Participaram deste estudo 44 crianças e adolescentes, regularmente matriculados em uma escola municipal na cidade de São Luís - MA, com idade entre 10 e 15 anos, sendo excluídos aqueles que haviam realizado cirurgias otognáticas prévias, estivessem fazendo ou já tivessem feito tratamentos ortodônticos, sofrido traumas na face e que não apresentassem o consentimento assinado pelos pais.

O diagnóstico de DTM foi avaliado com o eixo I do critério diagnóstico em pesquisa para disfunção temporomandibular RDC/TMD(5) e para avaliar o diagnóstico de DTM um avaliador previamente treinado para utilização dessa ferramenta. $\bigcirc$ paquímetro digital foi utilizado para avaliar a amplitude de movimento mandibular.

O RDC/TMD consiste na mensuração da amplitude de movimento mandibular, avaliação da dor à palpação, além da observação de estalidos e crepitações ao movimento. (6) Este mesmo avaliador questionou sobre os hábitos parafuncionais por meio das perguntas elaboradas no método de Merighi et al, 2007,,$^{(7)}$ por meio de perguntas como: Você tem o hábito de morder os lábios? Você tem o hábito de roer as unhas? Você tem o hábito de apertar os dentes? Você tem o hábito de ranger os dentes? Você tem o hábito de chupar o dedo?

Este projeto foi aprovado pelo comitê de ética em pesquisa da Universidade CEUMA, parecer 1.307.233. Todas as crianças assinaram o termo de assentimento e todos os pais e responsáveis assinaram o termo de Consentimento livre e esclarecido.

Para a análise inicialmente, os grupos foram divididos por gênero, as variáveis quantitativas foram descritas por média e desvio padrão (média \pm DP) e as qualitativas foram apresentadas em frequência. Os dados foram analisados no software Statistical Package for the Social Sciences (SPSS 18.0). A idade, peso e altura entre os grupos foram comparados por meio da análise de variância (ANOVA). As proporções entre os grupos foram comparadas utilizando-se o teste Qui-quadrado $\left(\chi^{2}\right)$ de correção de Yates. O Odds ratio (OR) e o intervalo de confiança (IC) foram usados para avaliar a associação entre o gênero e o diagnóstico de DTM. O nível de significância estatística de $\mathrm{p} \leq 0,05$ foi adotado. 


\section{RESULTADOS E DISCUSSÃO}

Quarenta e sete estudantes foram avaliados, três alunos foram excluídos por fazerem uso de aparelho ortodôntico. Do total de 44 indivíduos da amostra,59,09\% $(n=26)$ pertenciam ao gênero feminino, foi observada uma frequência de diagnóstico de 29,54\% $(n=13)$ de DTM miogênica, onde o gênero feminino apresentou maior frequência de diagnóstico 38,46\% $(n=10) p<0,05$. Foi observado risco quatro vezes maior de desenvolvimento de DTM no gênero feminino em relação ao masculino Odds ratio /IC 4,7 (2,22-9,93). (Tabela 1)
Nossos achados são semelhantes aos de Rubin et al., ${ }^{(7)}$ que avaliaram 153 crianças e adolescentes de ambos os gêneros e observaram frequência de DTM de 35\%. Ainda, Suvinem, et al. (2005),(8) também avaliaram 91 crianças de ambos os gêneros e observaram predominância do diagnóstico de DTM no sexo feminino.

Todos os sujeitos da amostra estudada, $100 \%$ apresentavam algum hábito parafuncional; os hábitos mais constatados foram roer unha, mascar chiclete, morder objetos, e os os ítens mais citados por ambos os gêneros foi mascar chiclete, feminino $(77,78 \% \mathrm{n}=14)$ e masculino $(92,31 \% \mathrm{n}=24)$. (Figura 1)

Tabela 1 - Valores médios e desvios padrões dos dados demográficos de idade, peso, altura, frequência de diagnóstico de DTM em porcentagens e chance de desenvolver DTM em relação ao sexo

\begin{tabular}{lccccc}
\hline \multicolumn{1}{c}{ Gênero } & Altura $\left(\mathrm{cm}^{2}\right)$ & Peso $(\mathrm{Kg})$ & Idade & Diagnóstico de DTM & Odds ratio/IC \\
\hline $\begin{array}{l}\text { Feminino } \\
59,09 \% \mathrm{n}=26\end{array}$ & $155 \pm 20$ & $53 \pm 5$ & $13,07 \pm 1,46$ & $38,46 \%{ }^{*} \mathrm{n}=10$ & $4,7(2,22-9,93)$ \\
Masculino & $150 \pm 30$ & $51 \pm 2$ & $12,76 \pm 1,5$ & $16,66 \% \mathrm{n}=3$ & \\
$40,90 \% \mathrm{n}=18$ & & & & & \\
\hline
\end{tabular}

* $\mathrm{p}=0,001 \mathrm{IC}=$ intervalo de confiança $\mathrm{OD}=$ Odds ratio

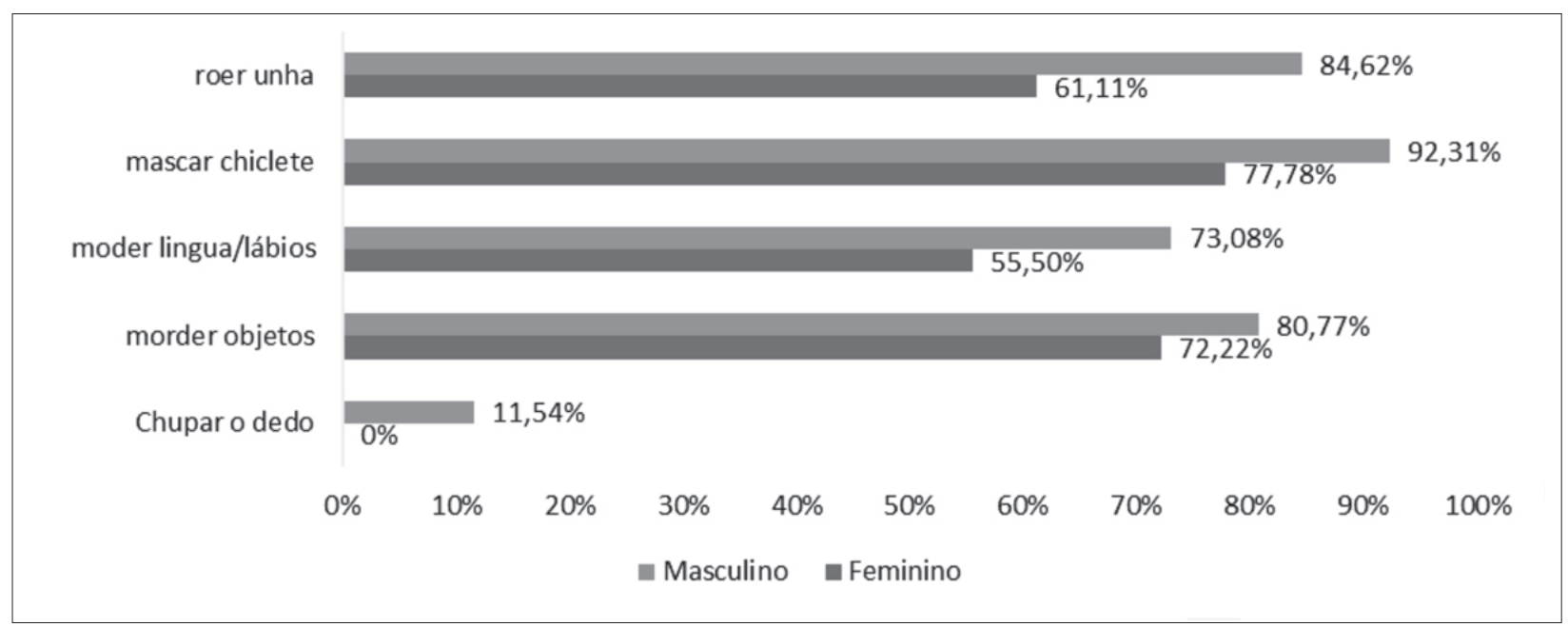

Figura 1. Frequência em porcentagem dos hábitos parafuncionais orais em crianças e adolescentes

Semelhante aos nossos achados, Rubin et al. ${ }^{(9)}$ também encontraram alta frequência, 93\%, de hábitos parafuncionais orais em sua amostra de crianças e adolescentes. Esses achados possibilitam estabelecer uma relação entre a prática de hábitos parafuncionais orais e a frequência de DTM.

Os hábitos parafuncionais orais promovem um desgaste e/ou mal posicionamento mandibular; roer unhas e marcar chiclete causam excesso de pressão em uma da face de mastigação causando efeitos deletérios, assim como o aumento de chances de desenvolvimento de DTM em níveis mais dolorosos.

Considerando que sinais e sintomas de DTM são muito comuns na população em geral, quando os primeiros sinais e sintomas aparecem pode ser de fato difícil identificar o começo do agravo e aparecimento da DTM. Além disso, devido à etiologia ser multifatorial, muitos sintomas podem ser confundidos ou menosprezados.

Assim, o aumento da frequência de hábitos parafuncionais orais podem ser usados como um preditor 
do surgimento da DTM. Corroborando com nossa hipótese, Mingheli et al. ${ }^{(10)}$ concluíram em seu estudo com crianças e adolescentes que hábitos parafuncionais aumentam a probabilidade do surgimento dessa doença da DTM.

Pesquisas com crianças apresentam dificuldades, como interesse em participar e a atenção durante os questionamentos, além da necessidade da autorização de terceiros, justificando o tamanho da amostra estudada e, dessa forma, os resultados expostos precisam ser analisados à luz de todo conhecimento disponível na literatura sobre esse assunto. As ferramentas validadas, principalmente o uso de uma ferramenta de diagnóstico como o RDC/TMD aplicada por um avaliador treinado, são considerados pontos fortes desta pesquisa.

\section{CONCLUSÃO}

A frequência de diagnósticos de DTM em crianças e adolescentes foi alta principalmente no gênero feminino (38\%), que possuem quatro vezes mais risco de desenvolver DTM quando comparados ao gênero masculino. Todos os alunos praticavam pelo menos um habito parafuncional oral e indivíduos do sexo. Como ainda existe carência de estudos, sobretudo com diagnóstico, de DTM em crianças e adolescentes, é necessário que este assunto continue sendo estudado, em amostras maiores uma vez que a DTM pode reduzir a qualidade de vida e o rendimento escolar, devido ao desconforto e/ou dores. Bem como o desenvolvimento de estudos de prevenção e promoção do conhecimento ainda na infância sobre os fatores que promovem o surgimento ou agravamento da DTM.

\section{REFERÊNCIAS}

1. American Society Of Temporomandibular Joint Surgeons (ASTJS). Guidelines for diagnosis and management of disorders involving the temporomandibular joint and related musculoskeletal structures. Cranio. 2003;21(1):68-76.

2. Pedroni CR, De Oliveira AS, Guaratini MI. Prevalence study and symptoms of temporomandibular disorders in university students. J Oral Rehabil. 2003 Mar;30(3):283-9.

3. Biasotto-Gonzalez DA. Abordagem interdisciplinar das disfunções temporomandibulares. São Paulo: Manole, 2005.

4. Bayardo RE, Mejia JJ, Orozco S, Montoya K. Etiology of oral habits. ASDC J Dent Child. 1996 Sep-Oct;63(5):350-3.

5. Dworkin SF, Leresche L. Research diagnostic criteria for temporomandibular disroders: review, criteria, examinations and specifications, critique. J Craniomandib Disord. 1992 Fall; 6(4):301-55.

Headache Medicine, 8, n.4, p.120-123, Oct./Nov./Dec. 2017
6. Chaves TC, Oliveira AS, Bevilaqua-Grossi D. Principais instrumentos para avaliação da disfunção temporomandibular (parte II): critérios diagnósticos; uma contribuição para a pratica clínica e de pesquisa. Fisioter. Pesqui. [Internet]. 2008; 15(1): 92-100.

7. Merighi LB, Silva MM, Ferreira AT, Genaro KF, Berretin-Felix G. Occurrence of temporomandibular disorder (TMD) and its relationship with harmful oral habits in children from Monte Negro - RO. Rev CEFAC 2007;9:497-503.

8. Suvinen TI 1, Reade PC, Kemppainen P, Könönen M, Dworkin SF. Review of etiological concepts of temporomandibular pain disorders: towards a biopsychosocial model for integration of physical disorder factors with psychological illness impact factors. Eur J Pain. 2005 Dec;9(6):613-33.

9. Friedman Rubin P, Erez A, Peretz B, Birenboim-Wilensky R, Winocur $E$. Prevalence of bruxism and temporomandibular disorders among orphans in southeast Uganda: A gender and age comparison. Cranio. 2017 May 30:1-7.

10. Minghelli B, Cardosol, Porfírio M, Gonçalves R, Cascalheiro $S$, Barreto $V$, et al. Prevalência de desordem temporomandibular em crianças e adolescentes de escolas públicas no sul de Portugal. N Am J Med Sci. 2014 Mar;6(3): 126-32.

Correspondência

Manoel Gomes de Araúio Neto netto_guerrerodecristo@hotmail.com

Recebido: 05 de outubro de 2017

Aceito: 30 de outubro de 2017 